\title{
The helical tomotherapy thread effect
}

\author{
M. W. Kissick ${ }^{\text {a) }}$ \\ Departments of Medical Physics and Engineering Physics, University of Wisconsin Medical School, \\ Madison, Wisconsin 53706-1532 \\ J. Fenwick \\ Departments of Human Oncology and Medical Physics, University of Wisconsin Medical School, Madison, \\ Wisconsin 53706-1532 \\ J. A. James \\ Department of Medical Physics, University of Wisconsin Medical School, Madison, Wisconsin 53706-1532 \\ R. Jeraj \\ Department of Medical Physics, University of Wisconsin Medical School, Madison, Wisconsin 53706-1532, \\ and TomoTherapy, Inc., Madison, Wisconsin 53717-1954
}

J. M. Kapatoes

TomoTherapy, Inc., Madison, WI 53717-1954

H. Keller

Princess Margaret Hospital, Toronto Ontario, Canada

T. R. Mackie

Departments of Medical Physics, Human Oncology, Biomedical Engineering, and Engineering Physics, University of Wisconsin Medical School, Madison, Wisconsin 53706-1532

G. Olivera

TomoTherapy, Inc., Madison, Wisconsin 53717-1954

E. T. Soisson

Department of Medical Physics, University of Wisconsin Medical School, Madison, Wisconsin 53706-1532

(Received 25 August 2004; revised 22 February 2005; accepted for publication 25 February 2005; published 27 April 2005)

\begin{abstract}
Inherent to helical tomotherapy is a dose variation pattern that manifests as a "ripple" (peak-totrough relative to the average). This ripple is the result of helical beam junctioning, completely unique to helical tomotherapy. Pitch is defined as in helical CT, the couch travel distance for a complete gantry rotation relative to the axial beam width at the axis of rotation. Without scattering or beam divergence, an analytical posing of the problem as a simple integral predicts minima near a pitch of $1 / n$ where $n$ is an integer. A convolution-superposition dose calculator (TomoTherapy, Inc.) included all the physics needed to explore the ripple magnitude versus pitch and beam width. The results of the dose calculator and some benchmark measurements demonstrate that the ripple has sharp minima near $p=0.86(1 / n)$. The 0.86 factor is empirical and caused by a beam junctioning of the off-axis dose profiles which differ from the axial profiles as well as a long scatter tail of the profiles at depth. For very strong intensity modulation, the 0.86 factor may vary. The authors propose choosing particular minima pitches or using a second delivery that starts 180 deg off-phase from the first to reduce these ripples: "Double threading." For current typical pitches and beam widths, however, this effect is small and not clinically important for most situations. Certain extremely large field or high pitch cases, however, may benefit from mitigation of this effect. (C) 2005 American Association of Physicists in Medicine. [DOI: 10.1118/1.1896453]
\end{abstract}

Key words: helical, tomotherapy, thread, dose uniformity

\section{INTRODUCTION}

Helical tomotherapy ${ }^{1}$ is an intensity modulated radiotherapy (IMRT) method of delivering therapeutic doses with a high degre of conformity. In contrast to standard radiotherapy linacs, the helical tomotherapy device (TomoTherapy, Inc., Madison, WI, USA) operates with a ring gantry that rotates at a constant speed around the patient. ${ }^{2}$ The couch translates inward concurrently at a constant speed. The result is a helical delivery pattern, unique to the field of radiotherapy with the geometry similar to a helical CT (computed tomography) scan device. Using a linear accelerator, tomotherapy can produce MVCT (megavoltage CT) images in addition to delivering treatment. ${ }^{3}$

In contrast to serial tomotherapy, ${ }^{4}$ helical tomotherapy does not have the same, nonhelical beam junctioning effects, but in this paper we will characterize a subtle type of "helical beam junctioning" occurring with helical tomotherapy. In addition, there is a beam divergence in the fan beam geometry used in helical tomotherapy that results in a complex off-axis dose profile for each helical rotation. This helical junctioning 


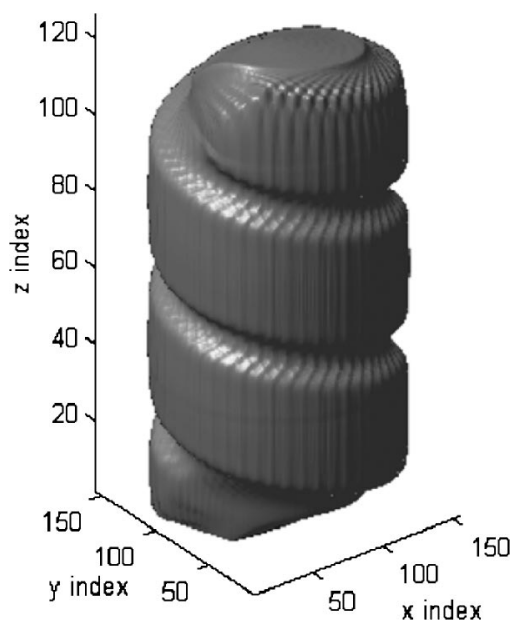

FIG. 1. An isodose at unity pitch (couch motion of one beam width for a full rotation) is displayed and calculated with the TomoTherapy, Inc. dose calculator. In the simulation used for this illustration, $161 \times 161 \times 144$ calculation space is used for this simulation. The transverse index scaling is $0.198 \mathrm{~cm} /$ index in both directions and the longitudinal scaling is $0.125 \mathrm{~cm} /$ index. The "thread effect" name is easy to see from this depiction. The isodose value is about $50 \%$ of the maximum, therefore near the edge of the exposed field near the edge of the phantom.

issue results in small dose delivery patterns known as the "thread effect." These patterns, shown as an isodose surface in Fig. 1 for an unmodulated wide field delivery on a coaxial circular cylindrical phantom, are often faintly visible on many delivery plan isodose plots and quality assurance (QA) films. The general purpose of this paper is to explain and introduce the origins of these helical patterns, also known as helical "ripples" and to propose methods to reduce the helical beam junctioning patterns.

In the Appendix, an analytical approach is presented to provide the context for understanding the results of some experimental data and a convolution-superposition dose calculator provided by TomoTherapy, Inc. The dose calculator code results are compared with a representative sample of measurements on the prototype tomotherapy device installed at the University of Wisconsin Hospital in the results section.

\section{METHODS}

\section{A. Geometry and problem setup}

For simplicity, this present study is restricted to dose behavior in a cylindrical geometry with a constant angular velocity source rotating about an axis coincident with the cylinder. The source also translates axially along the cylinder to model the couch movement at constant velocity. This simple geometry has direct relevance for quality assurance, dosimetry, and testing phantoms commonly used for tomotherapy. ${ }^{5}$ In the Appendix, the geometry and spatial variables are also pictorially defined. The source rotates at a distance, $r_{0}$ (fixed at $\mathrm{SAD}=85 \mathrm{~cm}$ for all systems in use to date), from the axis of symmetry. The phantom edge is at a distance $r=a$ in radius from the axis. We also define dimensionless variable $\rho$ $\equiv r / r_{0}$. The beam is from an approximate point source and so is divergent in both cone and fan beam directions. The beam is collimated, and for this study we assume that the beam covers the whole phantom in cross section. The important width for our problem is the width defined by the angle, $\alpha$, and this is small for tomotherapy for this study, $\sim 3^{\circ}$, hence a "fan beam." 1 The beam width, $w_{0}$, is defined as the longitudinal $(z)$ width of the beam at the central axis. A default beam width of $5 \mathrm{~cm}$ is used in the computer calculations and the measurements unless explicitly specified. Therefore, the beam is narrower closer to the source than $r_{0}$ and wider further from the source, and this divergence is a factor in the thread effect.

A key quantity is the pitch, defined as follows:

$$
p \equiv \frac{z_{0}}{w_{0}} .
$$

The numerator, $z_{0}$, above is the axial couch distance traveled for one gantry rotation. Therefore, since the rotational speed and axial translation speed are constant, the rotation angle at any point is simply related to the axial motion in general as

$$
\theta=2 \pi \frac{z}{w_{0} \cdot p}+\theta_{0}=2 \pi \frac{z}{z_{0}}+\theta_{0} .
$$

where $\theta_{0}$ is the initial start angle. In reality, the helical tomotherapy machine currently delivers a modulated intensity of this beam around 51 angles in each rotation. In this study, however, all the leaves are open and, therefore, no intensity modulation is performed. There is a well-defined helical symmetry. The position in angle is linearly related to the axial position, according to Eq. (2) above. In the absence of intensity modulation, the only difference from one transverse cross section to the next is the angular phase. All of the necessary fluence variation is contained in a single cross section, and therefore, the analytical description in the Appendix is two-dimensional.

Note that the relative ripple magnitude, $R(r)$, is defined as the peak-to-trough relative to the average as follows:

$$
R(r)=2 \frac{D(r)_{\max }-D(r)_{\min }}{D(r)_{\max }+D(r)_{\min }} .
$$

Where the dose, $D(r)$, is described in the Appendix for an idealized model. This ripple definition is just twice the average modulation of the dose variation at a given radius.

\section{B. Dose calculator numerical model}

TomoTherapy, Inc. (Madison, WI, USA) provided a stand-alone version of the Hi-Art treatment planning system for use in this study; the dose calculator provided is the latest release, and is called by the optimizer. In the stand alone version used here, the leaf sinograms were created by hand, simple because there is no modulation and, therefore, all intensity values are constant in a broad beam geometry.

The TomoTherapy, Inc. dose calculator ${ }^{6}$ is a convolutionsuperposition code that uses a newer and more accurate algorithm called "cumulative-cumulative collapsed-cone kernel (CCK)," see Ref. 6 more details. The CCK method utilizes the standard collapsed cone method of releasing en- 
ergy into cones of equal solid angle but also with enhanced accuracy for challenging situations where the electron fluence can vary within a voxel. The calculation is fully threedimensional (3D) with pencil beam ray-tracing for TERMA and Monte Carlo calculated kernels providing information on photon scattering and electron transport. A sample isodose plot of the dose calculator output in 3D is presented in Fig. 1. Because for this study, we have a homogeneous phantom and broad beam geometry, the resolution used for the calculations of $161 \times 161 \times 144$ is adequate.

\section{Experimental measurements}

To benchmark the dose calculator values, some values were determined experimentally with a $30 \mathrm{~cm}$ diameter solid water phantom with a mid-sagittal film view on the prototype tomotherapy device installed at the University of Wisconsin Hospital Comprehensive Cancer Center. This device has since been decommissioned and replaced with the new HiArt device. Some measurements were also taken on the new Hi-Art device. The beam energy spectrum has been found to be the same between these devices. Both are $6 \mathrm{MV}$ beams.

The films were scanned with a Vidar scanner and analyzed with rit113 software to plot cross-sectional dose to obtain ripple values (relative peak-to-trough values). The multileaf collimator leaves were left wide open without any modulation which matches the delivery sinogram used in the dose calculator above.

The relative value used for this study, the "ripple" defined by Eq. (3) was measured manually from profile plots from both the dose calculator and the measurements. The average dose reaches a maximum on-axis after one beam width has translated longitudinally, therefore, one needs several rotations at small pitches in order to accurately measure the ripple from the profiles. At larger pitches this can be challenging. In all experimental data, error bars represent maximum uncertainty as value is read from plots by rit113 software. The penumbra is about a half a centimeter wide with long scatter tails at depth. Therefore, the smallest fan beam width setting of $1.12 \mathrm{~cm}$ is dominated by penumbra. ${ }^{7} \mathrm{We}$ chose to perform the measurements at $4.98 \mathrm{~cm}$ beam width to minimize the penumbra effects and to maximize the thread effect. See Ref. 7 for details of the on-axis behavior of helical dose delivery and issues of beam width and scatter tails.

\section{Intensity modulated example and double thread}

A test case was devised to quickly investigate the results of this study. The target is imbedded within four avoidance structures. The TomoTherapy, Inc. optimizer and dose calculator compute the expected dose profiles. Films were then placed in a phantom and this test plan was delivered.

Figure 2 is an example of a complex test delivery pattern in which the proper choice of pitch produced better dose uniformity because of a decreased thread effect. Two treatment plans were generated for a cylindrically shaped solid water phantom, used for helical tomotherapy QA procedures. A pitch of $0.287(=0.86 / 3)$ was used for the first plan while
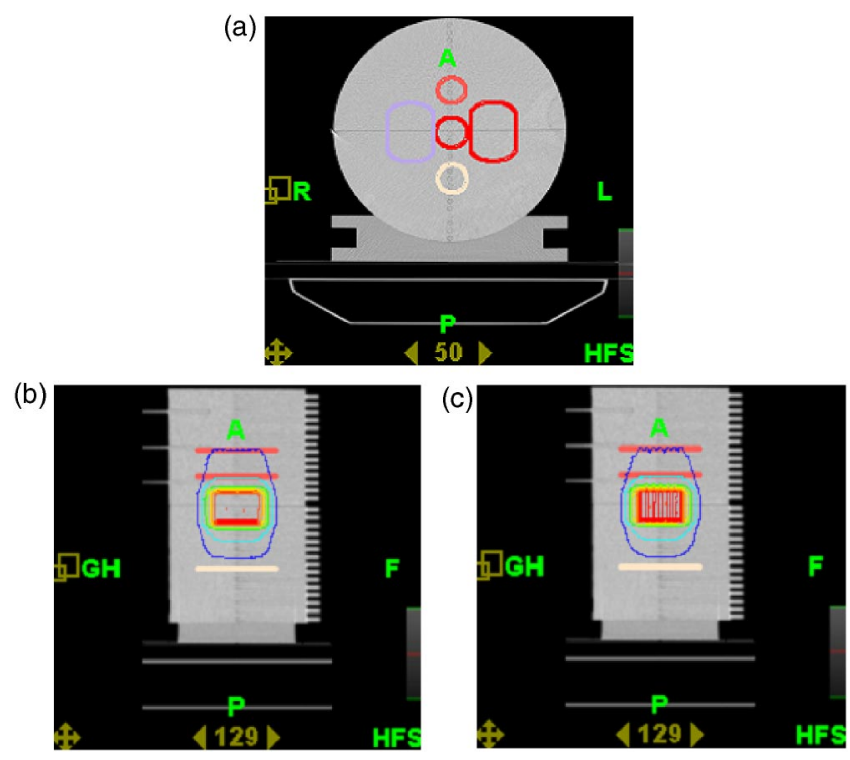

FIG. 2. An intensity modulated example with a solid water phantom $(32 \mathrm{~cm}$ transverse diameter, and $18 \mathrm{~cm}$ long), typical of QA procedures with helical tomotherapy. The plan is a central target surrounded by four avoidance structures in (a) the transverse view with the planning kVCT image with contoured regions-of-interest. In (b) and (c) the sagittal views are shown with the calculated dose contours for pitches of $0.287(=0.86 / 3)$ and 0.35 , respectively. One can easily see the reduction of the thread effect for the choice of pitch $=0.86 / 3$.

a pitch of 0.35 was used for the second plan. Structures included a centrally located cylindrical target structure that extended $5 \mathrm{~cm}$ in the superio-inferior direction, see Fig. 2(a). Avoidance structures were drawn directly anterior, posterior, right, and left of the target and given constraints to both simulate an actual treatment plan as well as increase the degree of modulation. The pitch was the only parameter that was varied between the two plans. All other aspects of the plan were identical. The chosen constraints resulted in a modulation factor of 2.05 for both plans. The modulation factor is the ratio of the maximum open time for a leaf delivering the maximum intensity to the mean open time. The larger the modulation factor, the more freedom the system has to provide the specified dose distributions, but the intensity profiles are more peaked. Each plan was prescribed to deliver 2 Gy per fraction to the target. After completion, the plans were treated on the Hi-Art Tomotherapy unit. The phantom was set up as planned and a film was placed in a coronal orientation at the center of the phantom for analysis. In another quick test, a film is left in place within the phantom in the same way as any other measurement, and another high pitch treatment is delivered except that the initial gantry position is $180 \mathrm{deg}$ shifted in phase angle to produce a "double threading" example.

\section{RESULTS}

\section{A. Minima pitch dependence}

Instead of finding the ripple minima at the values expected from the analytical approach described in the appendix at $p_{\min }=1 / n$, the TomoTherapy, Inc. dose calculator and 


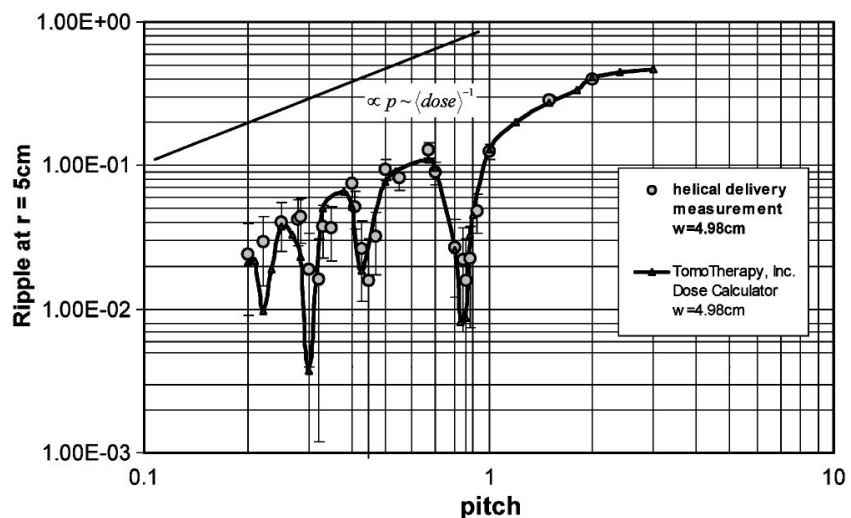

FIG. 3. The ripple (peak-to-trough relative to the average) calculated with the TomoTherapy, Inc. dose calculator compared with some measurements on a TomoTherapy machine with film in a cylindrical phantom. The sharp minima occur at $p_{\min } \approx 0.86 \cdot(1 / n)$ where $n$ is an integer. The overall trend for $p<1$ is related to the average dose increasing as the pitch decreases.

some representative measurements from the prototype TomoTherapy device at the University of Wisconsin Hospital both show minima at

$$
p_{\min }=(0.86 \pm 0.03) \cdot(1 / n) \text {. }
$$

The results in Fig. 3 demonstrate a very close agreement between theory and data, and the reader should note that the ripple [Eq. (3)] is a first-order quantity. There is some uncertainty in the exact values of these minima as these values are rapidly varying near the minima. The dose calculator gives the minima at $0.86,0.43,0.30$, and 0.22 , but the measurements give these minima at $0.86,0.45$, and 0.30 . Each of these minima may change within these uncertainties for situations that deviate from the ideal setup of this experiment, but this variation investigation is the subject of future work. As the beam width at isocenter, $w_{0}$, is varied, the dose calculator results indicate that the minima do not change their position, and the magnitude of the ripple, in general, below $p \sim 1$ is decreased due to the beam edge influence increasing as the beam width decreases (see Fig. 4).

Of course at $p>1$, one sees the expected leveling off of the ripple since the thread is so loose at large pitch that it simulates a static beam. In fact, as can be seen in Fig. 4, the width of $1.12 \mathrm{~cm}$ is so narrow that penumbra completely dominates the field, and its large pitch value also falls below the others. This observation is also presented in a related paper that concentrates entirely on the dose on axis. ${ }^{7}$ As the pitch is decreased below unity, the overall ripple decreases in proportion to the pitch. This is expected because the ripple in Eq. (3) is defined relative to the average dose value that is, in turn, inversely proportional to the pitch. The most interesting aspect of the plots of Figs. 3 and 4 are the particular values near $p_{\min } \approx 0.86 \cdot(1 / n)$. In the next sub-section, the origins of this factor are explained-it is the result of the divergence and intensity profiles of the fan-beam itself in addition to the scatter tail at depth evident in the dose profile.

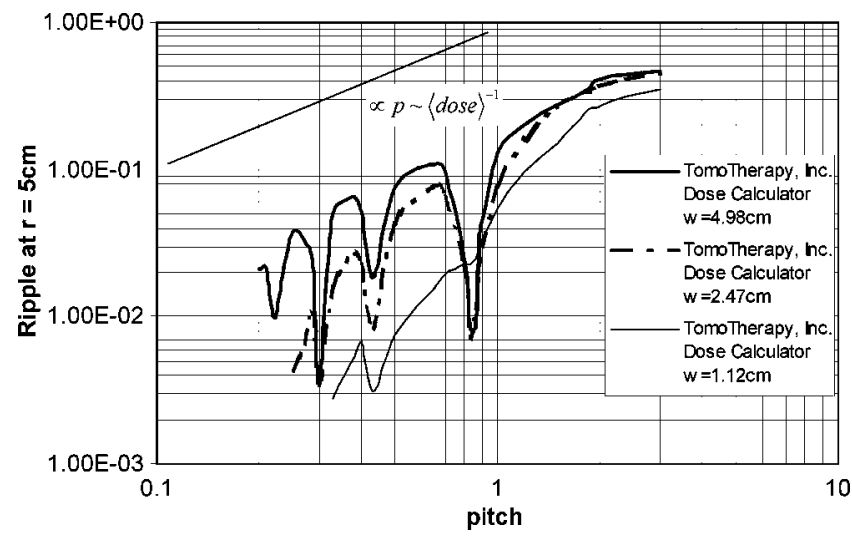

FIG. 4. The TomoTherapy, Inc. dose calculator ripple calculation (peak-totrough relative to the average) for three beam widths. The $2.47 \mathrm{~cm}$ beam width is most commonly used clinically. The $p_{\min } \approx 0.86 \cdot(1 / n)$ remains the same, but the overall ripple decreases with decreasing beam width. At 1.12 $\mathrm{cm}$ beam width, the dose profile is strongly dominated by scatter and beam edge effects.

\section{B. Helical junctioning-the $\mathbf{0 . 8 6}$ factor}

The dose calculator provided the flexibility to explore the helical dose delivery one rotation at a time. In fact, for a helical delivery pattern, the dose integral for a single complete rotation is a fundamental unit, and is all that is needed to reconstruct the entire continuous rotation. One can recreate a continuous rotational delivery by shifting $p \cdot w_{0}$ distance and adding multiple single rotation delivery dose profiles. If, for every radially off-axis dose profile plotted parallel to the rotation axis, there were no functional differences from the on-axis triangular shape profile (convolving a square with a square), then the minima would be expected to agree with the analytical approach described in the Appendix. However, in Fig. 5 one can see that the off-axis profiles are not straight lines. The reason for the complex shape off-axis in Fig. 5 is

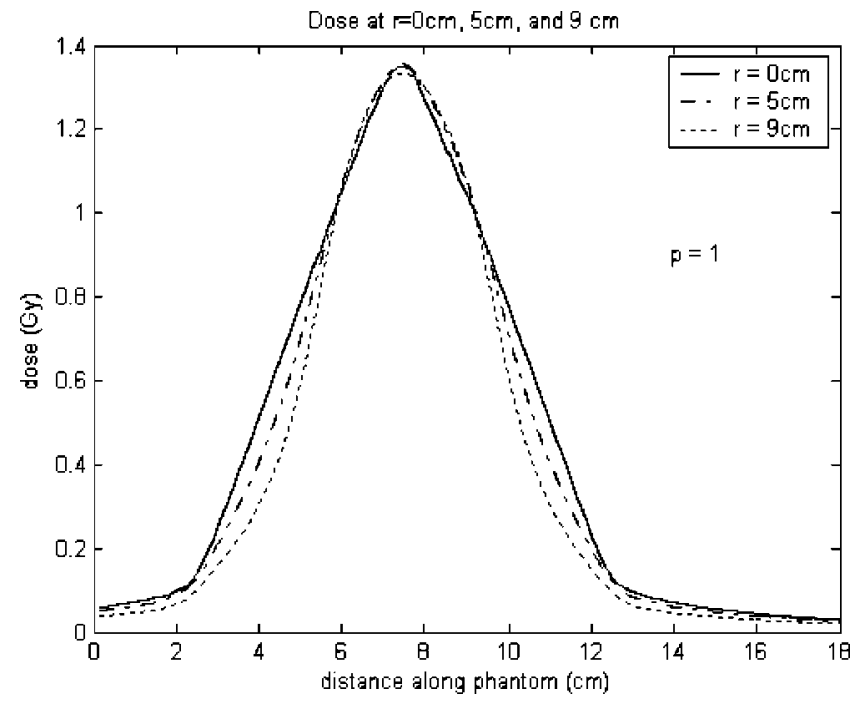

FIG. 5. Single rotation dose profile for unity pitch at radii $=0,5,9 \mathrm{~cm}$ out from the isocenter. Off-axis, the dose profile deviates from the triangular shape expected from the convolution of the beam width with itself that we see at $r=0 \mathrm{~cm}$. 
related to beam divergence. The integrated field strength grows faster than linear at the field edge because the field is weaker at either of the edges if on the far side from the source. If the field edge is on the near side closer to the source, then divergence causes the beam width to be smaller off-axis. Both effects lead to a reduced slope at the edges of the integrated field. At the center of the field, the source will at some point be closer and therefore the beam is stronger than if there were no beam divergence. All together, as the beam rotates, the complex shapes off-axis presented in Fig. 5 are produced. The consequence is that when the next rotation is added to this one, the off-axis profiles line up at a value of pitch less than $1 / n$. In Fig. 6, this point is graphically presented. The shift and add of a single rotation is performed for pitch $=1.0$ [Fig. 6(a)] and 0.86 [Fig. 6(b)] with the dose calculator. The shift and add is performed in three-dimensions with the full single rotation dose, and the resultant onedimensional profiles are extracted. One can see in Fig. 6 that the correct junctioning is accomplished not with pitch of unity, but occurs at about 0.86 [Fig. (b)]. In Fig. 6 at pitch $=0.7[$ Fig. 6(c)], there is overlap at the junction, and at pitch $=1.00$ [Fig. 6(a)], there is a gap at the junction. This is the explanation for the $p_{\min } \approx 0.86 \cdot(1 / n)$ minima seen in Figs. 3 and 4. Also notice in Figs. 5 and 6 that there is a long scatter tail at depth. Because the beams are narrow and this tail is long, the effective full width at half maximum (FWHM) where the rotations get matched is influenced by the tails of the neighboring rotations. This effect also contributes to the beam junctioning getting tighter which is a "tuned" junction to lower pitches: $p_{\min } \approx 0.86 \cdot(1 / n)$. At values of pitch corresponding to higher order minima ( $n=2,3,4, \ldots)$ the same junctioning effects occur except that it occurs with additional overlapping beams at each junction.

\section{Overall trend: Pitch $<1$}

The reader will also notice in all of the plots of ripple versus pitch that at pitches less than unity, there is a unity power law dependence on pitch: $R \sim p$. This is merely a consequence of the way that the relative ripple is defined. It is defined relative to the average dose. Therefore, since the slices overlap increasingly with decreasing pitch in a proportional manner, the pitch goes as the average dose at that radii: $p \sim\langle\text { dose }\rangle^{-1}$. As the pitch exceeds unity, this overlapping effect is no longer present and the relative ripple saturates at its high pitch value that is identical to the static beam value.

\section{Radial dependence}

Throughout the paper for consistency, the authors have concentrated on a radius $=5 \mathrm{~cm}$ in a cylindrical geometry. This choice allows for easy comparison between models and measurements. The ripple varies approximately linearly versus radius, see Figs. 7 and 8 . One can easily see that at the isocenter, there is no ripple at all. Of course at the isocenter there is complete circular symmetry.
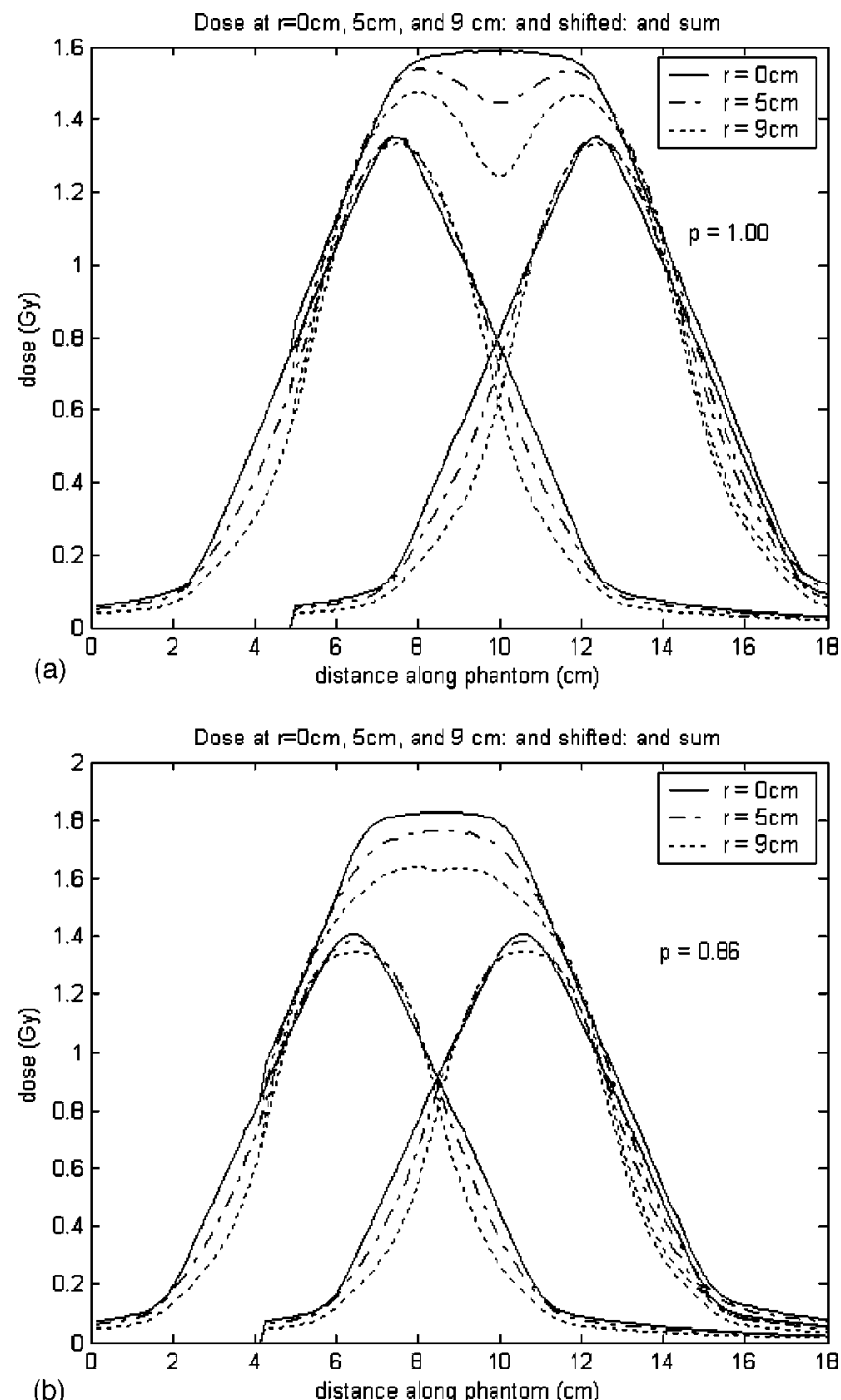

(b)

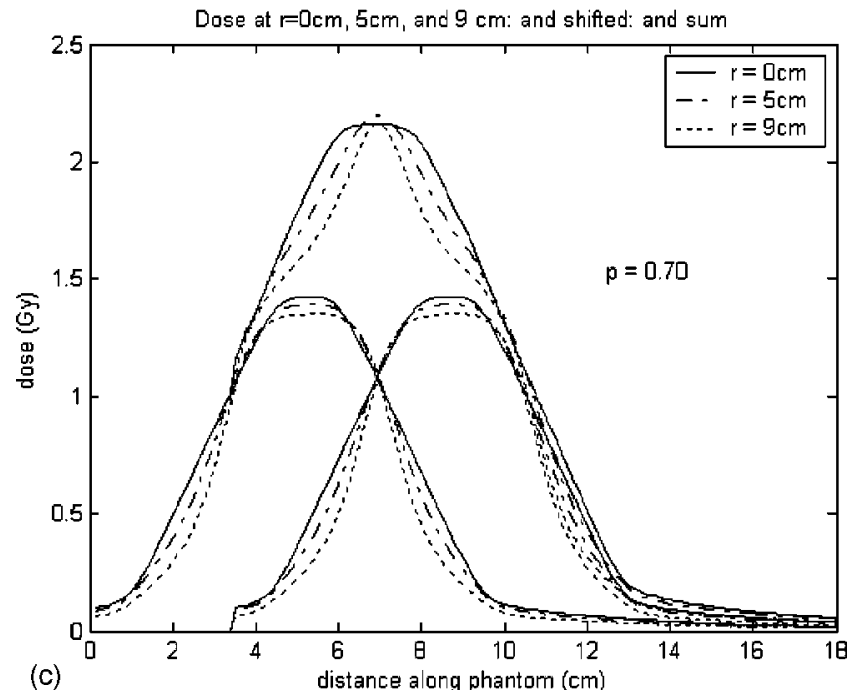

FIG. 6. Single rotation dose profile at radii $=0,5,9 \mathrm{~cm}$ out from the isocenter, then shifted by width $*$ pitch and added to the unshifted profiles. The shift and add is done with the full three-dimension dose profile. Notice the consequence of the non-triangular off-axis dose profiles shape and long axial dose profiles tails in creating the best junctioning at pitch of 0.86 (b) rather than at the expected pitch of 1.0 (a) or at pitch of 0.70 (c). 


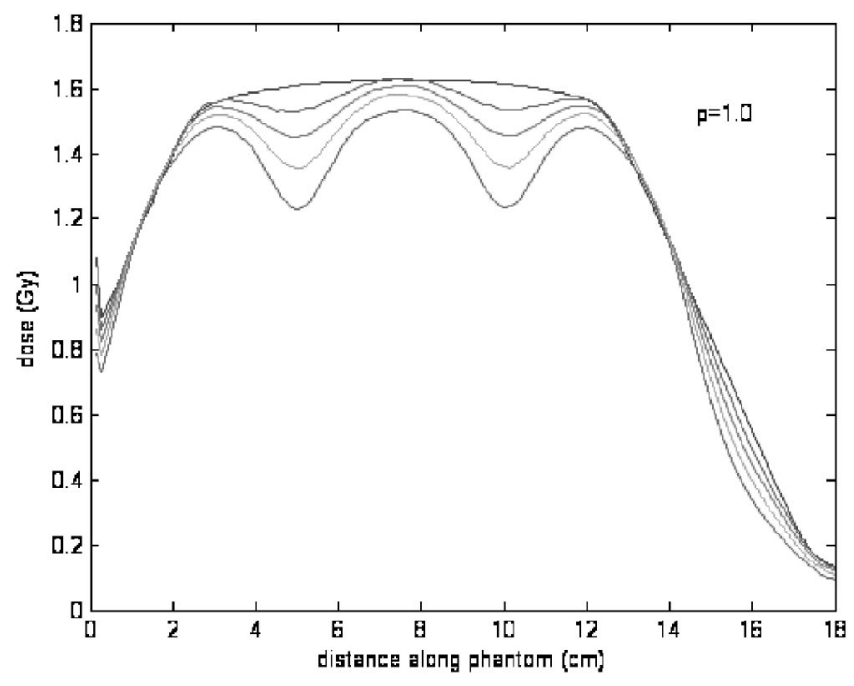

FIG. 7. Axial dose profiles for $r=0,3,5,7$, and $9 \mathrm{~cm}$ for continuous rotation at pitch of 1.0 from the dose calculator for an unmodulated beam. The largest ripple is for $r=9 \mathrm{~cm}$, and they diminish towards no ripple at $r$ $=0 \mathrm{~cm}$.

\section{E. Intensity modulated example}

An increased thread effect $(>10 \%)$ can clearly be seen upon visual inspection of the processed film for the intensity modulated example (see Sec. II D) treatment of the second plan in which the "bad" pitch of 0.35 was used, see Figs. 9(a) and 9(b). Analysis with the Tomotherapy software also shows decreased uniformity in the dose profile for the second plan. The thread effect also appears on axis in this figure, but this difference is likely due to the high degree of modulation used for the neighboring avoidance structures.

The degree to which the 0.86 factor varies for real situations with significant intensity modulation remains to be seen. There would certainly still be minima corresponding to helical junctioning such that every point gets approximately the same integral for a complete rotation, but strong and nonsymmetric intensity modulation might be expected to alter the single rotation profiles and scatter tail size. Nevertheless, one does expect a 0.86 factor in situations where a target gets radiation from most of the angles used in helical tomotherapy.

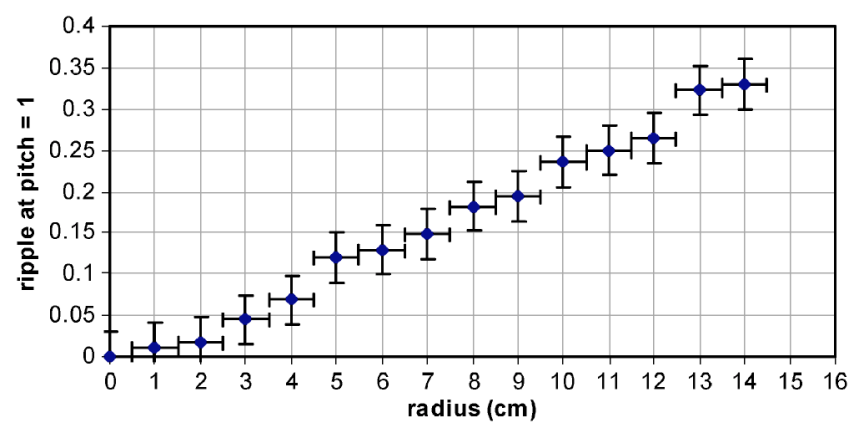

FIG. 8. The ripple for single threading at pitch=1.0 from the film data described in the text is representative of the result from any pitch that the relative ripple varies roughly linear vs radius with zero ripple at $r=0 \mathrm{~cm}$. The error bars represent the maximum uncertainty.

\section{DISCUSSION}

\section{A. Minima and helical junctioning}

There is a unique concept of "helical beam junctioning" presented here. There is no direct analogy with other devices. In the case of serial tomotherapy, the junctioning is at certain discrete transverse cross sections separated at each beam width. In this case, the junctioning can be made very smooth but potentially very rough. By contrast, for helical tomotherapy, the junctioning is in every surface-it is helical in nature. Because helical tomotherapy uses overlapping helices, this helical junctioning magnitude is always reduced in relative magnitude because of overlapping beams. The result is that even rough or "un-tuned" junctioning in tomotherapy is not at all severe. The typical beam width used clinically is $2.5 \mathrm{~cm}$ at a pitch less than 0.5 where the maximum thread effect magnitude is less than 3\% (see Fig. 4).

The 0.86 factor empirically arrived at in this investigation bespeaks an interesting concept of "effective pitch or width." From Eqs. (1) and (4),

$$
p_{\min }=0.86 \cdot \frac{z_{0}}{w_{0}} .
$$

Therefore, for the same axial displacement, $z_{0}$, we might say that there is an "effective pitch" with respect to off-axis beam helical junctioning:

$$
p_{\text {eff }}=(0.86)^{-1} p \text {. }
$$

In other words, a real pitch of 0.86 is an effective pitch of unity since it represents a "well-tuned" beam junctioning. One could equally as well conceive of an effectively smaller beam width by this factor. The obvious solution to minimize the thread effect is to only choose those pitches that minimize these ripples.

From Fig. 6 it is also apparent that the critical beam junctioning is approximately the same at all radii. In other words, the factor 0.86 is approximately the same for each radii and for each minima to within $3 \%$ for $0.2<p<1.0$ and at radii $<10 \mathrm{~cm}$. There is some uncertainty due to measurement technique and calculation parameters, but choosing any pitch near these values would be a big improvement over values that correspond to maximum ripple. For a real clinical situation, there may be more variation in this factor than presented in this study, and this variation is the subject of future work. The goal of this study is to make helical tomotherapy users aware of these minima and to provide some understanding about their origins.

\section{B. Double threading}

Initial exploration of a technique called "double threading" demonstrates a significant benefit in thread effect ripple reduction even at a very large pitch. The technique is to simply re-apply the same dose delivery again, but starting the gantry at 180 deg off-phase from the initial delivery. One result of this delivery scheme is to add additional minima (twice as many); see Appendix for an illustration. If a single thread, then all of the angles are sampled at $p=1 / n$, but if 


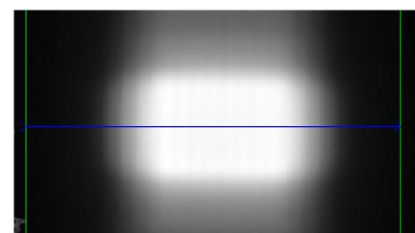

(a)

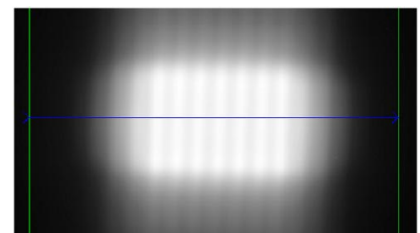

(b)

FIG. 9. An intensity modulated example result from Fig. 2 setup with a solid water phantom, typical of QA procedures with helical tomotherapy. In (a) and (b) are the film verifications of this treatment for pitches of 0.287 $=0.86 / 3$ ) and 0.35 , respectively. One can easily see the reduction of the thread effect for the choice of pitch $=0.86 / 3$.

double threaded, then there are additional minima at $p$ $=2 / n$, some of these are the same such as $2 / 2=1 / 1$. The major effect is to decrease the ripple for all pitches by an order of magnitude (see Appendix).

The salient point is illustrated in Fig. 10. In part a of this figure, one might conclude in general that a high pitch of 1.5 is clinically unusable because of the large ripple. However, with double threading [Fig. 10(b)], this pitch is clinically very feasible. Since pitch is proportional to the couch speed, there are potential large field or motion dynamics that could benefit from double threading.

\section{Clinical implications}

Future work will concentrate more on the clinical implications of the thread effect. Currently with the typical beam widths of $2.5 \mathrm{~cm}$ and typical pitches of less than 0.5, Fig. 4 indicates little concern even if nothing were done differently. However, for most cases, the use of pitch equaling 0.215 , $0.287,0.43[p=0.86 \cdot(1 / n)]$ would be much better for dose uniformity than $0.25,0.33,0.5(p=1 / n)$. See Figs. 2 and 9 for an example. Therefore, one should choose the particular ripple minima pitches whenever possible. There may be strong intensity modulated cases such as radiation delivered to only one side of the object. In such cases, the 0.86 factor may vary, and the resolution of this issue is reserved for future work.

For large pitch, the use of the double threading technique is recommended. One could presumably even further improve the dose uniformity with even more threads-similar to a wound cable.
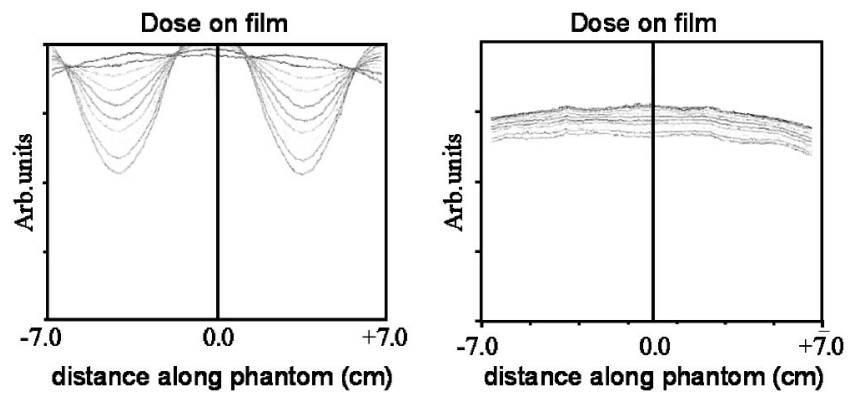

FIG. 10. Axial dose profiles for continuous rotations at pitch of 1.5 from single threading (a) vs double threading (b) for an unmodulated beam. These film profiles were produced with rit113 software.
Because the ripple increases with increasing distance offaxis, it behooves us to center the tumor at the isocenter if desirable. This could be done with programmed modulation for a single tumor. The difficulty occurs for multiple tumors within a single transverse cross section or slice. In this case, the use of pitch minima or double threading should be used.

Perhaps the development of a unique quality assurance protocol would benefit from the concept of effective pitch or effective width in terms of helical junctioning. Because these minima are so sharp in pitch, the ripple observation provides a sensitive measure of pitch. There may be some quality assurance procedures that would require the avoidance rather than enhancing these ripples.

\section{CONCLUSION}

The helical dose modulation or "ripple" that results from not junctioning the off-axis beam profiles in helical tomotherapy is small for typical clinical situations. However, one could "fine-tune" the delivery by choosing the pitches that minimize this ripple by junctioning the off-axis profiles: $p_{\text {min }} \approx 0.86 \cdot(1 / n)$. Future work will concentrate on clinical issue of strong intensity modulation to determine under what conditions the 0.86 factor varies.

We found that the off-axis dose profiles for each complete rotation have shapes that differ from the triangular axial profile. In addition, there are long scatter tails than extend deep into neighboring rotations. Taken together, one gets a complex junctioning between rotations that could lead us to another new idea- "effective pitch" or equivalently "effective beam width" for off-axis helical junctioning.

It remains unclear at this time what are the clinical consequences of helical junctioning. Certainly for most situations there are few consequences beyond just a deeper understanding of the dose delivery process for helical tomotherapy. Moreover, it is also certain that rare situations always arise clinically that stretch typical settings for any medical device. In a situation where large pitches are desired, double threading could be used to reduce the ripple to appropriate levels. Some quality assurance procedures that may be developed specifically for this machine could benefit from the implicit measurement of pitch that these ripples would provide.

\section{ACKNOWLEDGMENTS}

The authors are grateful for useful conversations with Dr. Susanta Hui and Dr. Ralf Hinderer. The staff and facilities of the University of Wisconsin Hospital and Clinics and Comprehensive Cancer Center were vital for the success of this project. Particularly vital was the cooperation with the staff and products of TomoTherapy, Inc. Dr. Mackie, Dr. Olivera, and Dr. Kapatoes have ownership interests in TomoTherapy Inc., which is commercializing helical tomotherapy. This work was made possible by a United States National Institute of Heath (NIH), United States National Cancer Institute Training Grant and NIH Grant (T32 CA09206 and PO1 CA88960). 


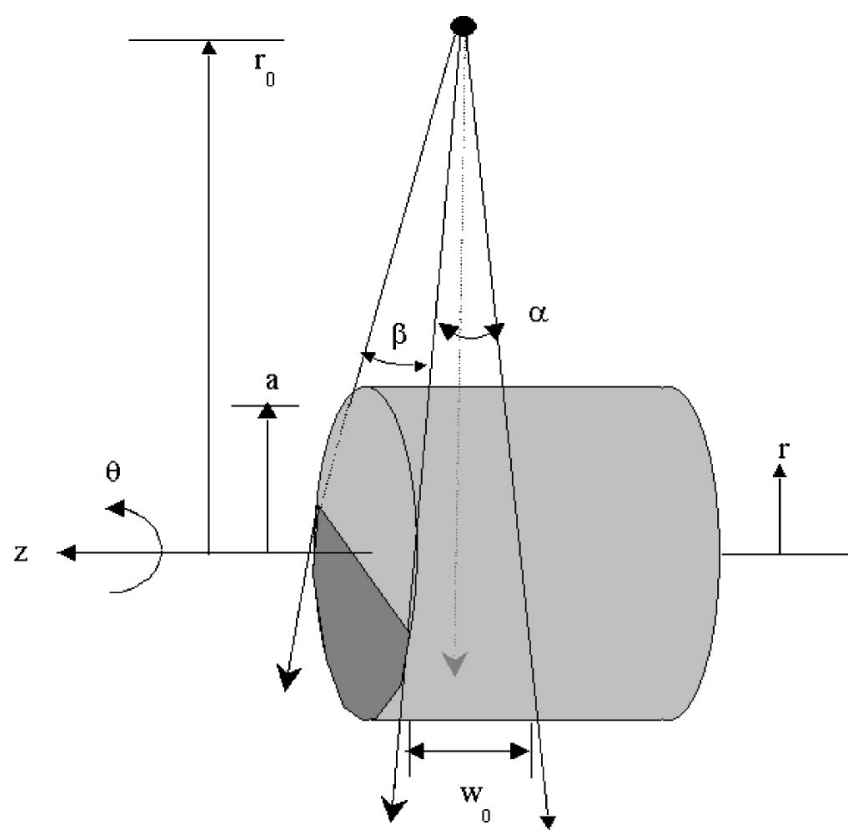

FIG. 11. A pictorial definition of the cylindrical geometry and basic parameters used for modeling. Notice that a realistic model of the beam shape needs to include its shape and correct angles. We restrict ourselves to a constant helical motion rotated around the axis of a long cylindrical water phantom.

\section{APPENDIX: NONDIVERGING BEAM MODEL}

In order to obtain an understanding of the thread effect, a simplistic model with a nondiverging beam is used and presented in this Appendix. It is possible to understand the problem analytically with no beam divergence. The problem is setup with the same definitions presented in Sec. II A and with Fig. 11.

\section{Analytical model foundation}

A helical integral is formulated as follows. The fluence is assumed to fall off in an inverse-square nature

$$
K(\rho, \theta) \equiv \frac{1}{\left(1+\rho^{2}-2 \rho \cos (\theta)\right)} .
$$

The function $K$ above is actually just the relative fluence function or a crude dose approximation for a general gantry angle, $\theta$, described by Eq. (2) and Fig. 11. There are other effects that can be considered in the model such as attenuation with a simple $\exp \{-\mu d\}$ model, where $d$ is the depth of the beam in the phantom and $\mu$ is the linear attenuation coefficient, but for this analytical illustration, attenuation was not included. Of course, the dose at the outer layer of the phantom is incorrect if buildup is not included. There is also a central maximum in the beam intensity towards the isocenter (due to the forward nature of high energy, $\mathrm{MeV}$, bremsstrahlung generation in a linac). This central maximum factor is approximately $[1+2|\sin (\theta)| \rho]$ for the helical tomotherapy machine, which has an unflattened beam, ${ }^{2}$ and this multiplicative factor that accounts for higher fluence when

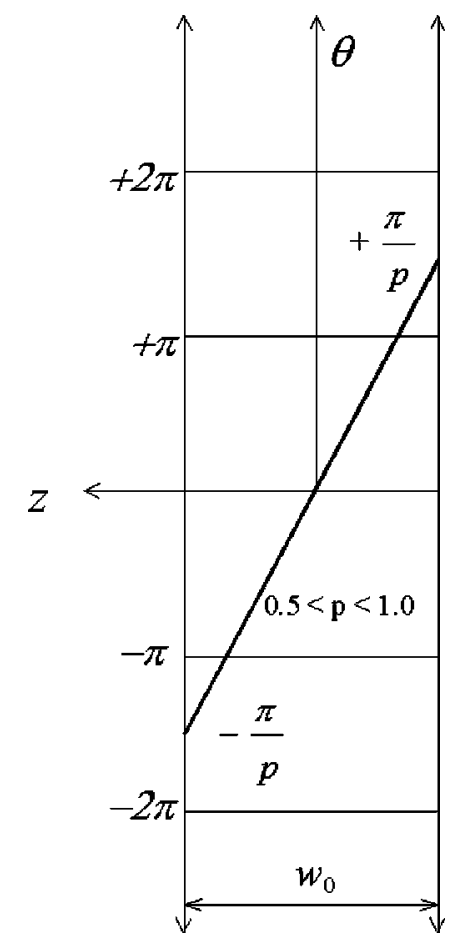

FIG. 12. The integral limits can be thought of as intersection points of the helical motion line with the beam width at a particular phase. Notice how this line changes slope relative to pitch.

the point is between the target and the isocenter is included as well.

The function $K^{\prime}$ is integrated in the following integral, the dose function in a cross section:

$$
D(r, \theta)=\int_{\theta_{-}}^{\theta_{+}} K^{\prime}(\rho, \theta-\xi) d \xi,
$$

where $K^{\prime}=K \times[1+2|\sin (\theta)| \rho]$. Here, much of the interesting physics is in the limit functions of the integral: $\theta_{ \pm}$. The dose is integrated between source positions where a voxel starts "seeing" the beam, $\theta_{-}$, and where its stops "seeing" the beam, $\theta_{+}$. The voxel view of the source movement is illustrated in Fig. 12. Note the important point that adding an arbitrary phase, $\theta_{0}$, to any $\theta$ in the above equations is the same as shifting along the axial direction according to Eq. (2).

In reality, there is beam divergence, and this can be handled as follows. Consider half of the cone-angle $\alpha, \gamma$ $=\alpha / 2$ : see Fig. 11 . The relationship between the angle limits and the beam divergence in the axial direction is found by trigonometry with the observation that

$$
\left|\frac{|\Delta z|-w_{0} / 2}{r \cos \left(\theta_{ \pm}\right)}\right|=\tan \gamma=\frac{w_{0} / 2}{r_{0}} .
$$

Half the couch travel distance during which the beam is visible to the point at $r, \Delta z$, is related to half of the angle range sampled by that point through the pitch relation of Eq. (2) 


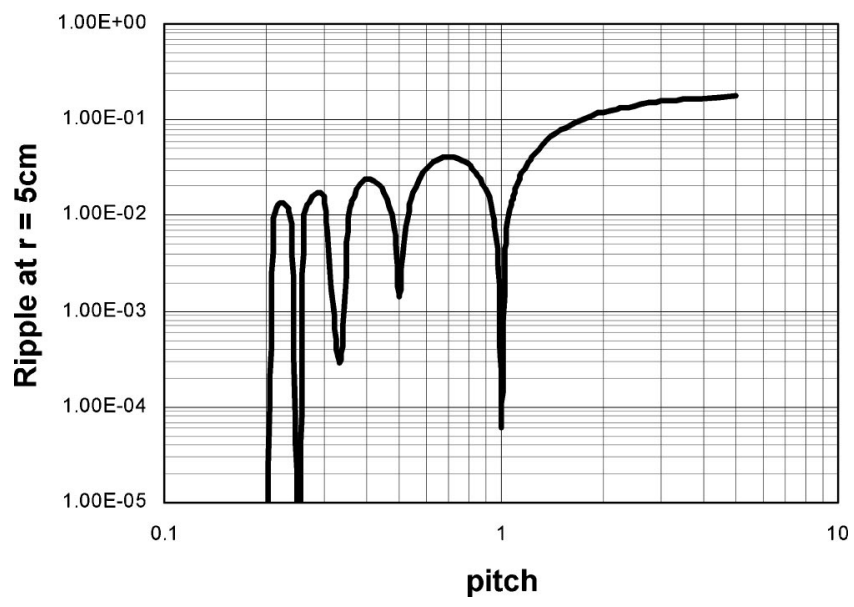

FIG. 13. A logarithmic plot of the relative ripple vs pitch for the case of a parallel beam [limits of Eq. (A6)]. Notice for single delivery, there are minima at pitch $=1 / n$ where $n$ is a positive integer.

$$
\theta_{ \pm}-\theta= \pm 2 \pi \frac{\Delta z}{w_{0} \cdot p}
$$

The angle $\theta$ is the angle where the dose is being solved. Combining the above equations to eliminate $\Delta z$ leads to, The limit functions are in general for a diverging beam

$$
\theta_{ \pm}=\theta \pm \frac{\pi}{p}\left[1+\rho \cos \left(\theta_{ \pm}\right)\right]
$$

It is interesting that pitch, radius, and source distance contain all of the needed geometry in this case with the beam width implicitly included. The obvious problem is that in Eq. (A1), the divergence width projection contains the angle limits being solved for. This leads to the relation above. Unfortunately, this function is transcendental and, therefore, impossible to handle analytically. Fortunately, a first-order approximation is possible because of the fan-beam geometry such that $\rho \equiv r / r_{0} \ll 1$. This approximation simulates an infinite source to axis (SAD) distance, $r_{0}$. It also implies a constant beam width at all radii and at all angles of $w_{0}$, a "rectangular beam." In this case, the limit functions are

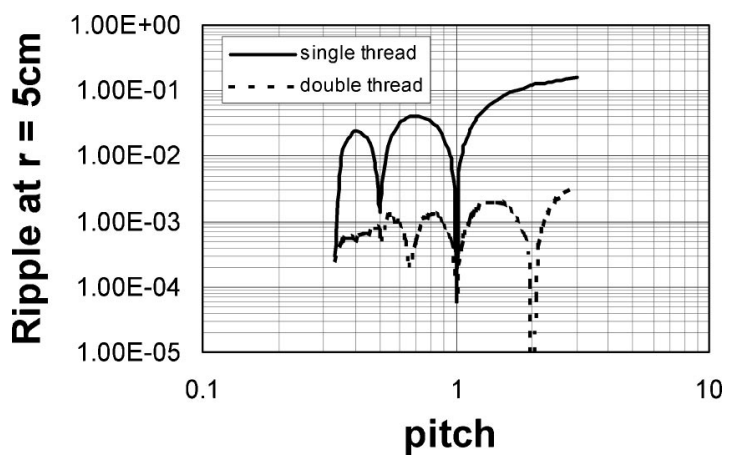

FIG. 14. The ripple for single threading versus double threading from the analytical model described in the text. Notice the additional minima for the double threading case.

$$
\theta_{ \pm}=\theta \pm \frac{\pi}{p}
$$

A pictorial view of these limits is provided in Fig. 12 where the path of a point happens to cross where $\theta_{ \pm} \sim \pm 3 \pi / 2$.

Missing from Fig. 11 is the depiction of the unflattened forward intensity enhancement due to tomotherapy's unflattened high energy bremsstrahlung directed at the isocenter. This intensity profile does affect the off-axis beam profile shape as well, but has a little role in the production of the thread effect.

\section{Analytical model results}

Regardless of the $K$ function, something very interesting happens in the solution of Eq. (A2) at certain pitches. If the pitch is $1 / n$ where $n$ is an integer, then, for a rectangular beam, $D(r, \theta)=D(r)$, and there is no ripple. For example, at $p=1$, the limits cover all possible angles exactly once for every point. In general, the integral spans over all unique values of $K$ exactly $n$ times. Hence, there is no ripple or thread effect. At pitches not at these values, the ripple is due to gaps or overlaps in beam coverage. The solution of Eq. (A2) using only Eqs. (A6) is plotted in Fig. 13. A simple Simpsons-rule numerical integration scheme is used to perform the integral in Eq. (A2).

We can see from Fig. 13 that there is a finite ripple value at $p \neq 1 / n$. The magnitude does generally decrease proportionally with decreasing pitch [since the ripple is defined relative to the average in Eq. (3)] with the exception of very local minima. At really large pitch values, the relative ripple saturates and is completely determined by the $K$ variation since the threads are independent of each other at $p \geqslant 2$.

\section{Double thread analytical illustration}

In Fig. 14, the analytical model is used to demonstrate the basic trend. It is expected that a similar picture would hold true for the dose calculator and real measurement results, but shifted by a factor of 0.86 . The Simpsons-rule integration that produced the values of the minima in Figs. 13 and 14 are susceptible to error accumulation. The minima are actually zero in value, and as the number of calculations increase for each point, the less deep the minima can become because of error accumulation. As the pitch decreases, more angles are integrated. Therefore, the high pitch minima are very deep (see the $p=2$ minima in Fig. 14). In addition, the low pitch minima are deeper and/or narrower in general for reasons more complex.

\footnotetext{
${ }^{a)}$ Electronic mail: mwkissick@wisc.edu

${ }^{1}$ T. R. Mackie, T. Holmes, S. Swerdloff, P. Reckwerdt, J. O. Deasy, J. Yang, B. Paliwal, and T. Kinsella, "Tomotherapy: A new concept for the delivery of dynamic conformal radiotherapy," Med. Phys. 20, 1709-1719 (1993).

${ }^{2}$ T. R. Mackie, J. Balog, K. Ruchala, D. Shepard, S. Aldridge, E. Fitchard, P. Reckwerdt, G. Olivera, T. Shui, and M. Mehta, "Tomotherapy," Semin Radiat. Oncol. 9, 108-117 (1999).

${ }^{3}$ K. J. Ruchala, G. H. Olivera, E. A. Schlosser, and T. R. Mackie, "Megavoltage CT on a Tomotherapy system," Phys. Med. Biol. 44, 2597-2621
} 
(1999).

${ }^{4}$ M. P. Carol, "Peacock: A system for planning and rotational delivery of intensity-modulated fields," Int. J. Imaging Syst. Technol. 6, 56-61 (1995).

${ }^{5}$ B. Paliwal, W. Tomé, S. Richardson, and T. R. Mackie, “A spiral phantom for IMRT and tomotherapy treatment delivery verification," Med. Phys. 29, 2503-2507 (2000).
${ }^{6}$ W. Lu, G. H. Olivera, M.-L. Chen, P. J. Reckwerdt, and T. R. Mackie, "Accurate convolution / superposition for multi-resolution dose calculations using cumulative tabulated kernels," Phys. Med. Biol. 50, 655-680 (2005).

${ }^{7}$ J. Fenwick, W. Tomé, M. Kissick, and T. R. Mackie, "Modelling simple helically delivered dose-distribution," Phys. Med. Biol. 50, 1505-1517 (2005). 